\title{
ON ALMOST COINCIDENCE POINTS IN GENERALIZED CONVEX SPACES
}

ZORAN D. MITROVIĆ

Received 19 April 2006; Accepted 7 June 2006

We prove an almost coincidence point theorem in generalized convex spaces. As an application, we derive a result on the existence of a maximal element and an almost coincidence point theorem in hyperconvex spaces. The results of this paper generalize some known results in the literature.

Copyright (C) 2006 Zoran D. Mitrović. This is an open access article distributed under the Creative Commons Attribution License, which permits unrestricted use, distribution, and reproduction in any medium, provided the original work is properly cited.

\section{Introduction and preliminaries}

The notion of a generalized convex space we work with in this paper was introduced by Park and Kim in [10]. In generalized convex spaces, many results on fixed points, coincidence points, equilibrium problems, variational inequalities, continuous selections, saddle points, and others have been obtained, see, for example, $[6,8,10-13]$.

In this paper, we obtain an almost coincidence point theorem in generalized convex spaces. Some applications to the existence of a maximal element of an almost fixed point theorem in hyperconvex spaces are given.

A multimap or map $F: X \multimap Y$ is a function from a set $X$ into the power set of a set $Y$. For $A \subset X$, let $F(A)=\bigcup\{F x: x \in A\}$. For any $B \subset Y$, the lower inverse and upper inverse of $B$ under $F$ are defined by

$$
\begin{aligned}
& F^{-}(B)=\{x \in X: F x \cap B \neq \varnothing\}, \\
& F^{+}(B)=\{x \in X: F x \subset B\},
\end{aligned}
$$

respectively. The lower inverse of $F: X \multimap Y$ is the map $F^{-}: Y \multimap X$ defined by $x \in F^{-} y$ if and only if $y \in F x$.

A map $F: X \multimap Y$ is upper (lower) semicontinuous on $X$ if and only if for every open $V \subset Y$, the set $F^{+}(V)\left(F^{-}(V)\right)$ is open. A map $F: X \multimap Y$ is continuous if and only if it is upper and lower semicontinuous. 
For a nonempty subset $D$ of $X$, let $\langle D\rangle$ denote the set of all nonempty finite subsets of $D$. Let $\Delta_{n}$ denote the standard $n$-simplex with vertices $e_{1}, e_{2}, \ldots, e_{n+1}$, where $e_{i}$ is the $i$ th unit vector in $\mathbb{R}^{n+1}$.

A generalized convex space or $G$-convex space $(X, D ; \Gamma)$ consists of a topological space $X$, a nonempty set $D$, and a function $\Gamma:\langle D\rangle \multimap X$ with nonempty values such that for each $A \in\langle D\rangle$ with $|A|=n+1$, there exists a continuous function $\varphi_{A}: \Delta_{n} \rightarrow \Gamma(A)$, such that $\varphi_{A}\left(\Delta_{J}\right) \subset \Gamma(J)$, where $\Delta_{J}$ denote the faces of $\Delta_{n}$ corresponding to $J \in\langle A\rangle$.

Particular forms of $G$-convex space are convex subsets of a topological vector space, Lassonde's convex space, a metric space with Michael's convex structure, $S$-contractible space, $H$-space, Komiya's convex space, Bielawski's simplicial convexity, Joó's pseudoconvex space, see, for example, [11-13].

For each $A \in\langle D\rangle$, we may write $\Gamma(A)=\Gamma_{A}$. Note that $\Gamma_{A}$ does not need to contain $A$. For $(X, D ; \Gamma)$, a subset $C$ of $X$ is said to be $G$-convex if for each $A \in\langle D\rangle, A \subset C$ implies $\Gamma_{A} \subset C$. If $D=X$, then $(X, D ; \Gamma)$ will be denoted by $(X, \Gamma)$. The $G$-convex hull of $K$, denoted by $G-\operatorname{co}(K)$, is the set

$$
\bigcap\{B \subset X: B \text { is a } G \text {-convex subset of } X \text { containing } K\} \text {. }
$$

Let $C$ be a $G$-convex subset of $X$, a map $F: C \multimap X$ is called $G$-quasiconvex if

$$
F(d) \cap S \neq \varnothing \quad \text { for each } d \in D \Longrightarrow F(u) \cap S \neq \varnothing \quad \text { for each } u \in \Gamma_{D} \text {, }
$$

for each $D \in\langle C\rangle$, and for each $G$-convex subset $S$ of $X$. If $X$ is a topological vector space and $\Gamma_{A}=\operatorname{co} A$, we obtain the class of quasiconvex maps, see, for example, [7, page 18].

Let $C$ be a subset of $X$, a map $F: C \multimap X$ is called $G$-KKM map if $\Gamma_{A} \subset F(A)$ for each $A \in\langle C\rangle$.

The following version of G-KKM-type theorem, see, for example, [13, page 49], will be used to prove the main result of this paper.

Theorem 1.1. Let $(X, \Gamma)$ be a G-convex space, $K$ a nonempty subset of $X$, and $H: K \multimap X a$ map with closed (open) values and G-KKM map. Then $\bigcap_{x \in D} H(x) \neq \varnothing$ for each $D \in\langle K\rangle$.

\section{Almost-like coincidence point theorem}

Theorem 2.1. Let $(X, \Gamma)$ be a G-convex space, $K$ a nonempty subset of $X, U$ a nonempty closed (open) G-convex subset of $X$, and $\mu: K \times K \multimap X$ a map such that

(1) for each fixed $y \in K$, the map $x \mapsto \mu(x, y)$ is upper (lower) semicontinuous map,

(2) for each fixed $x \in K$, the map $y \mapsto \mu(x, y)$ is G-quasiconvex map,

(3) there exists a set $D \in\langle K\rangle$ such that $\Gamma_{D} \subseteq K$ and $\mu(x, D) \cap U \neq \varnothing$ for each $x \in K$.

Then there exists $x_{U} \in K$ such that

$$
\mu\left(x_{U}, x_{U}\right) \cap U \neq \varnothing .
$$

Proof. Let for every $y \in K, H: K \multimap K$ be defined by

$$
H(y)=\{x \in K: \mu(x, y) \cap U=\varnothing\} .
$$


From assumption (1), we obtain that $H(y)$ is closed (open) set for each $y \in K$. We can prove that $H$ is not a G-KKM map. Namely,

$$
\bigcap_{y \in D} H(y)=\{x \in K: \mu(x, D) \cap U=\varnothing\}
$$

and from assumption (3), we obtain that

$$
\bigcap_{y \in D} H(y)=\varnothing
$$

So, by Theorem 1.1, $H: K \multimap K$ is not a $G$-KKM map. This implies that there exists $A \in$ $\langle D\rangle$ such that

$$
\Gamma_{A} \nsubseteq H(A)
$$

and hence there is an $x_{U} \in \Gamma_{A}$ such that $x_{U} \notin H(A)$. This implies that

$$
\mu\left(x_{U}, a\right) \cap U \neq \varnothing \quad \text { for each } a \in A .
$$

From assumption (2), we obtain

$$
\mu\left(x_{U}, x_{U}\right) \cap U \neq \varnothing .
$$

From Theorem 2.1, we have the following almost coincidence point theorem for topological vector space.

Theorem 2.2. Let $X$ be a topological vector space, $K$ a nonempty subset of $X, U$ a nonempty open (closed) convex neighborhood of 0 in $X$, and $F_{1}: K \multimap X, F_{2}: K \multimap X\left(F_{2}: K \rightarrow X\right)$ are maps such that

(1) the map $F_{1}$ is lower (upper) semicontinuous map with convex values,

(2) the map $F_{2}$ is quasiconvex,

(3) there exists a set $D \in\langle K\rangle$ such that $\operatorname{co} D \subseteq K$ and $F_{1}(x) \cap\left(F_{2}(D)+U\right) \neq \varnothing$ for each $x \in K$.

Then there exists $x_{U} \in K$ such that

$$
F_{1}\left(x_{U}\right) \cap\left(F_{2}\left(x_{U}\right)+U\right) \neq \varnothing .
$$

Proof. Taking $\mu(x, y)=F_{1}(x)-F_{2}(y)$ and $\Gamma_{A}=\operatorname{co} A$ in Theorem 2.1, we get the proof.

As an application of Theorem 2.2, we obtain the following result of existence of almost fixed point of Park [9, Theorem 2.1].

Corollary 2.3. Let $X$ be a topological vector space, $K$ a nonempty subset of $X, U$ a nonempty open (closed) convex neighborhood of 0 in $X$, and $F: K \multimap X$ a lower (upper) semicontinuous map with convex values such that there exists a set $D \in\langle K\rangle$ such that $\operatorname{co} D \subseteq K$ and $F(x) \cap(D+U) \neq \varnothing$ for each $x \in K$. Then there exists $x_{U} \in K$ such that

$$
F\left(x_{U}\right) \cap\left(x_{U}+U\right) \neq \varnothing .
$$


4 On almost coincidence points

Remark 2.4. The assumption

$$
F(x) \cap(D+U) \neq \varnothing, \quad \text { for each } x \in K,
$$

in Corollary 2.3 can be replaced by the following condition:

$$
F(X) \subseteq D+U
$$

In this case, we obtain the result of Kim and Park [4, Theorem 1.2].

\section{Almost coincidence point theorem in metrizable $G$-convex spaces}

Let $(X, \Gamma)$ be a metrizable $G$-convex space with metric $d$. For any nonnegative real number $r$ and any subset $A$ of $X$, we define

$$
B(A, r)=\bigcup\{B(a, r): a \in A\}
$$

where $B(a, r)=\{x \in X: d(a, x)<r\}$.

Similarly, we define

$$
B[A, r]=\bigcup\{B[a, r]: a \in A\}
$$

where $B[a, r]=\{x \in X: d(a, x) \leq r\}$.

In this case, we obtain the following result.

Theorem 3.1. Let $(X, \Gamma)$ be a metrizable $G$-convex space, $K$ a nonempty subset of $X, F_{1}$ : $K \multimap X$ a map with $G$-convex values, and $F_{2}: K \multimap X$ a map such that

(1) the map $F_{1}$ is lower semicontinuous,

(2) there exists a $\lambda \geq 1$ such that $G-\operatorname{co}\left(B\left(F_{2}^{-}(A), r\right)\right) \subseteq F_{2}^{-}(B(A, \lambda r))$, for all $G$-convex subsets $A$ of $X$ and nonnegative real number $r$,

(3) there exists a set $D \in\langle K\rangle$ such that $\Gamma_{D} \subseteq K$ and $F_{1}(x) \cap B\left(F_{2}(D), \varepsilon\right) \neq \varnothing$ for each $x \in K$, where $\varepsilon>0$.

Then there exists $x_{\varepsilon} \in K$ such that

$$
F_{1}\left(x_{\varepsilon}\right) \cap B\left(F_{2}\left(x_{\varepsilon}\right), \lambda \varepsilon\right) \neq \varnothing .
$$

Proof. Let for every $y \in K, H: K \multimap K$ be defined by

$$
H(y)=\left\{x \in K: F_{1}(x) \cap B\left(F_{2}(y), \varepsilon\right)=\varnothing\right\}
$$

From assumption (1), we obtain that $H(y)$ is open for each $y \in K$, further, from assumption (3), we obtain that

$$
\bigcap_{y \in D} H(y)=\varnothing
$$


So, by Theorem 1.1, $H: K \multimap K$ is not a G-KKM map. This implies that there exists $A \in$ $\langle D\rangle$ such that

$$
\Gamma_{A} \nsubseteq H(A)
$$

and hence there is an $x_{\varepsilon} \in \Gamma_{A}$ such that

$$
F_{1}\left(x_{\varepsilon}\right) \cap B\left(F_{2}(a), \varepsilon\right) \neq \varnothing \quad \text { for each } a \in A .
$$

Hence, we obtain

$$
F_{2}(a) \cap B\left(F_{1}\left(x_{\varepsilon}\right), \varepsilon\right) \neq \varnothing \quad \text { for each } a \in A .
$$

So, from assumption (2), we have

$$
F_{2}\left(x_{\varepsilon}\right) \cap B\left(F_{1}\left(x_{\varepsilon}\right), \lambda \varepsilon\right) \neq \varnothing,
$$

that is,

$$
F_{1}\left(x_{U}\right) \cap B\left(F_{2}\left(x_{U}\right), \lambda \varepsilon\right) \neq \varnothing
$$

Note that if in Theorem 3.1 a map $F_{2}(x)=\{x\}, x \in K$, and open balls are replaced by closed balls, we obtain following result.

Theorem 3.2. Let $(X, \Gamma)$ be a metrizable G-convex space, $K$ a nonempty subset of $X, F$ : $K \multimap X$ an upper semicontinuous map with $G$-convex values, and there exists a $\lambda \geq 1$ such that $G-\operatorname{co} B[A, r] \subseteq B[A, \lambda r]$, for all $G$-convex subsets $A$ of $X$ and nonnegative real number $r$. If there exists a set $D \in\langle K\rangle$ such that $\Gamma_{D} \subseteq K$ and $F(x) \cap B[D, \varepsilon] \neq \varnothing$ for each $x \in K$, where $\varepsilon>0$, then there exists $x_{\varepsilon} \in K$ such that

$$
F\left(x_{\varepsilon}\right) \cap B\left[x_{\varepsilon}, \lambda \varepsilon\right] \neq \varnothing .
$$

Corollary 3.3. Let $X$ be a metrizable $G$-convex space, $K$ a nonempty subset of $X, f: K \rightarrow$ $X$ a continuous map, and there exists a $\lambda \geq 1$ such that $G-\operatorname{co} B[A, r] \subseteq B[A, \lambda r]$, for all $G$-convex subsets $A$ of $X$ and nonnegative real number $r$. If there exists a set $D \in\langle K\rangle$ such that $\operatorname{co} D \subseteq K$ and $f(K) \subseteq B[D, \varepsilon] \neq \varnothing$, where $\varepsilon>0$, then there exists $x_{\varepsilon} \in K$ such that

$$
f\left(x_{\varepsilon}\right) \in B\left[x_{\varepsilon}, \lambda \varepsilon\right] .
$$

Corollary 3.4. Let $X$ be a metrizable G-convex space, $K$ a nonempty $G$-convex compact subset of $X, f: K \rightarrow K$ a continuous map, and there exists a $\lambda \geq 1$ such that $G-\operatorname{co} B[A, r] \subseteq$ $B[A, \lambda r]$, for all $G$-convex subsets $A$ of $X$ and nonnegative real number $r$. Then there exists $x \in K$ such that $f(x)=x$.

Remark 3.5. (1) Note that if $X$ is locally $G$-convex space, see, for example, [13, page 190], set $K$ is a compact set and $F: K \multimap K$ is map with closed values, from Theorem 3.2 we obtain a famous Fan-Glicksberg-type fixed point theorem. 
(2) If $X$ is a normed space, then Corollary 3.3 reduces to the result of Kim and Park [4, Theorem 2.1].

(3) Note that from Corollary 3.4, we obtain famous Schauder fixed point theorem.

Example 3.6. Let $X$ be a hyperconvex metric space, see, for example, [2, 3]. For a nonempty bounded subset $A$ of $X$, put

$$
\operatorname{co} A=\bigcap\{B: B \text { is closed ball in } X \text { containing } A\} .
$$

Let $\mathscr{A}(X)=\{A \subset X: A=\operatorname{co} A\}$. The elements of $\mathscr{A}(X)$ are called admissible subsets of $X$. It is known that any hyperconvex metric space $(X, d)$ is a $G$-convex space $(X, \Gamma)$, with $\Gamma_{A}=\operatorname{co} A$ for each $A \in\langle X\rangle$.

The $B(A, r)$ of an admissible subset $A$ of a hyperconvex metric space is also an admissible set, see [2, Lemma 4.10]. Let $F_{2}: K \multimap X$ be a $G$-quasiconvex map, that is, $F_{2}^{-}(A)$ is an admissible set for each admissible subset $A$ of $X$. Then the map $F_{2}$ satisfies the condition (2) in Theorem 3.1 for each real number $\lambda$ such that $\lambda \geq 1$.

From Theorem 3.1, we have the following almost coincidence point theorem and almost fixed point theorem in hyperconvex metric spaces.

Theorem 3.7. Let $X$ be a hyperconvex metric space, $K$ a nonempty subset of $X, F_{1}: K \multimap X$ a map with admissible values, and $F_{2}: K \multimap X$ a map such that

(1) the map $F_{1}$ is lower semicontinuous,

(2) the map $F_{2}$ is quasiconvex,

(3) there exists a set $D \in\langle K\rangle$ such that $\operatorname{co} D \subseteq K$ and $F_{1}(x) \cap B\left(F_{2}(D), \varepsilon\right) \neq \varnothing$ for each $x \in K$, where $\varepsilon>0$.

Then there exists $x_{\varepsilon} \in K$ such that

$$
F_{1}\left(x_{\varepsilon}\right) \cap B\left(F_{2}\left(x_{\varepsilon}\right), \varepsilon\right) \neq \varnothing .
$$

Note that if $K$ is a bounded set and $\alpha(\cdot)$ is a measure of noncompactness, then for each $\varepsilon>0$, there exists a finite set $D \subseteq K$ such that $K \subseteq B[D, \alpha(K)+\varepsilon)]$. In this case, lower semicontinuous map can be replaced by upper semicontinuous map.

Theorem 3.8. Let $X$ be a hyperconvex metric space, $K$ a nonempty bounded admissible subset of $X, F: K \multimap B[K, \mu]$ an upper semicontinuous map with admissible values, where $\mu>0$. Then for each $\varepsilon>0$, there exists $x_{\varepsilon} \in K$ such that

$$
x_{\varepsilon} \in B\left[F\left(x_{\varepsilon}\right), \alpha(K)+\varepsilon+\mu\right] .
$$

If in Theorem 3.8 set $K$ is a compact set and map $F$ with closed values, then as an immediate consequence, we obtain the result of existence of fixed point of Kirk and Shin [5, Corollary 3.5].

Finally, we obtain the result of existence of maximal elements for hyperconvex metric spaces.

Let $F: K \rightarrow 2^{X}$, where $2^{X}$ denotes the set of all subsets of $X$. An element $x \in K$ is a maximal element of $K$ if $F(x)=\varnothing$, see, for example, [1, page 33]. The $F$-maximal set of $F$ is defined as $M_{F}=\{x \in K: F(x)=\varnothing\}$. 
Corollary 3.9. Let $X$ be a hyperconvex metric space, $K$ a nonempty subset of $X, F_{1}: K \rightarrow$ $2^{X}$ a map with admissible values, and $F_{2}: K \rightarrow 2^{X}$ a map such that

(1) the map $F_{1}$ is lower semicontinuous,

(2) the map $F_{2}$ is quasiconvex,

(3) there exists a set $D \in\langle K\rangle$ such that $\operatorname{co} D \subseteq K$ and $F_{1}(x) \cap B\left(F_{2}(D), \varepsilon\right) \neq \varnothing$ for each $x \in K$, where $\varepsilon>0$.

If $x \notin F_{1}^{-}\left(B\left(F_{2}(x), \varepsilon\right)\right)$ for each $x \in K$, then $M_{F_{1}} \cup M_{F_{2}}$ is a nonempty set.

Corollary 3.10. Let $X$ be a hyperconvex metric space, $K$ a nonempty bounded admissible subset of $X, F: K \rightarrow 2^{X}$ an upper semicontinuous map with admissible values, and let $\varepsilon>$ 0 such that $x \in F^{-}(B[K, \varepsilon]) \backslash F^{-}(B[x, \alpha(K)+\varepsilon])$ for each $x \in K$. Then $F$ has a maximal element.

\section{Acknowledgment}

The author would like to thank the referee for his suggestions.

\section{References}

[1] K. C. Border, Fixed Point Theorems with Applications to Economics and Game Theory, Cambridge University Press, Cambridge, 1985.

[2] R. Espínola and M. A. Khamsi, Introduction to Hyperconvex Spaces, Kluwer Academic, Dordrecht, 2001.

[3] M. A. Khamsi, KKM and Ky Fan theorems in hyperconvex metric spaces, Journal of Mathematical Analysis and Applications 204 (1996), no. 1,298-306.

[4] I.-S. Kim and S. Park, Almost fixed point theorems of the Fort type, Indian Journal of Pure and Applied Mathematics 34 (2003), no. 5, 765-771.

[5] W. A. Kirk and S. S. Shin, Fixed point theorems in hyperconvex spaces, Houston Journal of Mathematics 23 (1997), no. 1, 175-188.

[6] L.-J. Lin, Applications of a fixed point theorem in G-convex space, Nonlinear Analysis 46 (2001), no. 5, 601-608.

[7] K. Nikodem, K-Convex and K-Concave Set-Valued Functions, Politechnika, Lodzka, 1989.

[8] S. Park, Continuous selection theorems in generalized convex spaces, Numerical Functional Analysis and Optimization 20 (1999), no. 5-6, 567-583.

[9] - Remarks on fixed point theorems for new classes of multimaps, Journal of the Academy of Natural Sciences, Republic of Korea 43 (2004), 1-20.

[10] S. Park and H. Kim, Admissible classes of multifunction on generalized convex spaces, Proceedings of College Nature Science, Seoul National University 18 (1993), 1-21.

[11] _ Coincidence theorems for admissible multifunctions on generalized convex spaces, Journal of Mathematical Analysis and Applications 197 (1996), no. 1, 173-187.

[12] Foundations of the KKM theory on generalized convex spaces, Journal of Mathematical Analysis and Applications 209 (1997), no. 2, 551-571.

[13] G. X.-Z. Yuan, KKM Theory and Applications in Nonlinear Analysis, Monographs and Textbooks in Pure and Applied Mathematics, vol. 218, Marcel Dekker, New York, 1999.

Zoran D. Mitrović: Faculty of Electrical Engineering, University of Banja Luka, Patre 5,

Banja Luka 78000, Bosnia and Herzegovina

E-mail address: zmitrovic@etfbl.net 